\title{
Problem on extremal decomposition of the complex plane
}

\author{
Iryna Denega and Yaroslav Zabolotnii
}

\begin{abstract}
In geometric function theory of a complex variable problems on extremal decomposition with free poles on the unit circle are well known. One of such problem is the problem on maximum of the functional

$$
r^{\gamma}\left(B_{0}, 0\right) \prod_{k=1}^{n} r\left(B_{k}, a_{k}\right),
$$

where $B_{0}, B_{1}, B_{2}, \ldots, B_{n}, n \geq 2$, are pairwise disjoint domains in $\overline{\mathbb{C}}$, $a_{0}=0,\left|a_{k}\right|=1, k=\overline{1, n}$ and $\gamma \in(0, n], r(B, a)$ is the inner radius of the domain $B \subset \overline{\mathbb{C}}$, with respect to a point $a \in B$. In the paper we consider a more general problem in which restrictions on the geometry of the location of points $a_{k}, k=\overline{1, n}$ are weakened.
\end{abstract}

In the geometric theory of univalent functions there are a number of papers and books devoted to the problems on non-overlapping domains. These problems were developed by B. Riemann [1], L. Bieberbach [2], H. Grötzsch [3], O. Teichmüller [4], M.A. Lavrentiev [5], G.M. Goluzin [6], James A. Jenkins [7], Z. Nehari [8], N.A. Lebedev [9], V. Hayman [10], M. Shiffer [11], R. Kühnau $[12]$ and others. A summary of this results in this sphere is contained in monographs $[13,14]$. In the works of these scientists the theory of univalent and multivalent functions of complex variable was created. The tasks of complex analysis and its application have predetermined development this direction.

Key Words: Inner radius of the domain, non-overlapping domains, $n$-radial system of points, separating transformation, quadratic differential, Green's function.

2010 Mathematics Subject Classification: Primary 30C75.

Received: 30.01.2018

Accepted: 30.03 .2018 
However, in spite of a significant amount of research, a number of difficult problems in the theory of extremal problems on non-overlapping domains are not solved. The development of methods and approaches to their solution is relevant.

In 1934 Lavrentev [5] solved the problem of product of conformal radii of two mutually non-overlapping simply connected domains. It was the first result of this direction. Lavrentev used this result to some aerodynamics problems. Goluzin in [6] generalized this problem in the case of an arbitrary finite number of mutually disjoint domains and obtained an accurate evaluation for the case of three domains. Further Kuzmina [15] showed that the problem of the evaluation for the case of four domains is reduced to the smallest capacity problems in the certain continuum family and received the exact inequality for four domains. For five or more areas full solution of the problem is not obtained at this time. Since, the evaluation of the product of conformal radii of mutually non-overlapping domains if $n \geq 5$ without any restriction on the domains $B_{k}$ and points $a_{k}, k=1, \ldots, 5$ is quite difficult and interesting problem. In 1975 Lebedev [9] considered the more general extremal problem of product of conformal radii.

Problem 1. [9] There are $n$ various fixed points $a_{k}, k=\overline{1, n}, n>3$, on a plane $w$. Functions $w=f_{k}(z), k=\overline{1, n}$, are regular in the circle $|z|<1$ and univalent map circle $|z|<1$ onto non-overlapping domains $B_{k}$, which contain the corresponding points $a_{k}, k=\overline{1, n}$, and in such a way, that $f_{k}(0)=a_{k}$, $k=\overline{1, n}$. What about maximum of product

$$
\prod_{k=1}^{n}\left|f_{k}^{\prime}(0)\right|^{\gamma_{k}} \longrightarrow \max , \quad \gamma_{k}>0, n>3
$$

relatively to any functions $f_{k}(z), k=\overline{1, n}$ ?

However, this problem is generally not solved so far. This problem was generalized to more general classes of multiply connected domains replacing conformal radius to the inner radius.

Until the mid 70-ies in the most works the point $z=0$, when displaying by the functions, passed into some different, but fixed points $a_{k}, k=\overline{1, n}$ of the complex plane. In 1968 Tamrazov [16] put forward the idea, that we can provide to the points $a_{k}, k=\overline{1, n}$, some "freedom". Such problems are called problems with "free" poles. We consider the problems where part of the poles are fixed, and some are free and which are called problems of "mixed" type.

Let $\mathbb{N}$ and $\mathbb{R}$ be the sets of natural and real numbers, respectively, let $\mathbb{C}$ be the complex plane, and let $\overline{\mathbb{C}}=\mathbb{C} \bigcup\{\infty\}$ be its one-point compactification, $\mathbb{R}^{+}=(0, \infty)$. Let $\chi(t)=\frac{1}{2}\left(t+t^{-1}\right), t \in \mathbb{R}^{+}$. Let $r(B, a)$ be the inner radius of the domain $B \subset \overline{\mathbb{C}}$ at the point $a \in B$ (see, e.g., $[13,14,17]$ ). Inner radius 
of the domain $B$ is associated with a generalized Green's function $g_{B}(z, a)$ of the domain $B$ by the relations

$$
\begin{gathered}
g_{B}(z, a)=\ln \frac{1}{|z-a|}+\ln r(B, a)+o(1), \quad z \rightarrow a . \\
g_{B}(z, \infty)=\ln |z|+\ln r(B, \infty)+o(1), \quad z \rightarrow \infty
\end{gathered}
$$

Note that to describe the extremal configurations of domains we use notion of quadratic differential (see, for example, [7, 18]). Quadratic differential $G(z) d z^{2}$ on a Riemann surface is a rule which associates to each local parameter $z$ mapping a parametric neighbourhood $U \subset \mathbb{R}$ into the extended complex plane $\overline{\mathbb{C}}(z: U \rightarrow \overline{\mathbb{C}})$, a function $G_{z}: z(U) \rightarrow \overline{\mathbb{C}}$ such that for any local parameters $z_{1}: U_{1} \rightarrow \overline{\mathbb{C}}$ and $z_{2}: U_{2} \rightarrow \overline{\mathbb{C}}$ with $U_{1} \cap U_{2}$ non-empty, the following holds in this intersection

$$
\frac{G_{z_{2}}\left(z_{2}(p)\right)}{G_{z_{1}}\left(z_{1}(p)\right)}=\left(\frac{d z_{1}(p)}{d z_{2}(p)}\right)^{2}, \quad p \in U_{1} \cap U_{2},
$$

here $z(U)$ is the image of $U$ in $\overline{\mathbb{C}}$ under $z$. In other words, a quadratic differential is a non-linear differential of type $(2,0)$ on a Riemann surface. The functions entering into the definition of a quadratic differential are ordinarily assumed to be measurable or even analytic.

Consider one open an extremal problem which was formulated in [17] in the list of unsolved problems and then repeated in monograph [14].

Problem 2. Consider the product

$$
I_{n}(\gamma)=r^{\gamma}\left(B_{0}, 0\right) \prod_{k=1}^{n} r\left(B_{k}, a_{k}\right)
$$

where $B_{0}, B_{1}, \ldots, B_{n}(n \geq 2)$ are pairwise disjoint domains in $\overline{\mathbb{C}}, a_{0}=0$, $\left|a_{k}\right|=1, k=\overline{1, n}$ and $0<\gamma \leq n$. Show that it attains its maximum at $a$ configuration of domains $B_{k}$ and points $a_{k}$ possessing rotational n-symmetry.

Presently, this problem is not completely solved, its solutions for certain particular cases are only known. In [17] the problem was solved for any $n \geq 2$ and $\gamma=1$. In paper [19] this problem was solved for any natural $n \geq 5$ and $0<\gamma \leq n$ but for condition $\alpha_{k} \leq \frac{2}{\sqrt{\gamma}}$, where $\alpha_{k}:=\frac{1}{\pi} \arg \frac{a_{k+1}}{a_{k}}, k=\overline{1, n}$. In [20] the Problem 2 was solved for $n \geq 5$ and $0<\gamma \leq \sqrt[4]{n}$. In [21] the solution was obtained for $n \geq 5$ and $0<\gamma \leq \sqrt[3]{n}$. In [23, 24] - for $n \geq 126$ and $0<\gamma \leq \sqrt{n}$

In monograph [13] the method of control functionals was proposed that allows us weaken the requirements on the geometry of the location of points 
systems. Thus we summarized the Problem 2 that is, instead of the unit circle we consider $n$-radial system of points satisfying certain conditions.

Let $n \in \mathbb{N}, n \geq 2$. A set of points $A_{n}:=\left\{a_{k} \in \mathbb{C}: k=\overline{1, n}\right\}$ is called $n$-radial system if $\left|a_{k}\right| \in \mathbb{R}^{+}, k=\overline{1, n}$, and

$$
0=\arg a_{1}<\arg a_{2}<\ldots<\arg a_{n}<2 \pi .
$$

We denote

$$
\begin{gathered}
\Gamma_{k}=\Gamma_{k}\left(A_{n}\right):=\left\{w: \arg a_{k}<\arg w<\arg a_{k+1}\right\}, \quad a_{n+1}:=a_{1}, \\
\alpha_{k}:=\frac{1}{\pi} \arg \frac{a_{k+1}}{a_{k}}, \quad \alpha_{n+1}:=\alpha_{1}, \quad k=\overline{1, n}, \quad \sum_{k=1}^{n} \alpha_{k}=2 .
\end{gathered}
$$

For an arbitrary $n$-radial system of points $A_{n}=\left\{a_{k}\right\}_{k=1}^{n}$ and $\gamma \in \mathbb{R}^{+} \cup\{0\}$ we assume

$$
\mathcal{K}^{(\gamma)}\left(A_{n}\right):=\prod_{k=1}^{n}\left[\chi\left(\left|\frac{a_{k}}{a_{k+1}}\right|^{\frac{1}{2 \alpha_{k}}}\right)\right]^{1-\frac{1}{2} \gamma \alpha_{k}^{2}} \prod_{k=1}^{n}\left|a_{k}\right|^{1+\frac{1}{4} \gamma\left(\alpha_{k}+\alpha_{k-1}\right)} .
$$

It is clear that the class of $n$-radial systems of points for which $\mathcal{K}^{(\gamma)}\left(A_{n}\right)=$ 1 automatically includes all systems of $n$ distinct points that are located on the unit circle.

We obtained result which shows the limits of the application of the method proposed in [25]. The following theorem characterizes the extremal domains if $0<\gamma \leq n^{\delta}$.

Theorem 1. For an arbitrary $\frac{1}{3}<\delta<\frac{2}{3}$ there is a natural $n_{0}$ such that for $n \geq n_{0}$ and $0<\gamma \leq n^{\delta}$ the following inequality holds

$$
r^{\gamma}\left(B_{0}, 0\right) \prod_{k=1}^{n} r\left(B_{k}, a_{k}\right) \leq r^{\gamma}\left(D_{0}, 0\right) \prod_{k=1}^{n} r\left(D_{k}, d_{k}\right)
$$

where $A_{n}=\left\{a_{k}\right\}_{k=1}^{n}$ is any $n$-radial system of points such that $\mathcal{K}^{(\gamma)}\left(A_{n}\right) \leq$ $1, \mathcal{K}^{(0)}\left(A_{n}\right) \leq 1, B_{k}, k=\overline{0, n}$, is any system of pairwise non-overlapping domains, $a_{k} \in B_{k} \subset \overline{\mathbb{C}}, a_{0}=0 \in B_{0} \subset \overline{\mathbb{C}}$. And equality in inequality (1) is attained, for example, if $a_{k}=d_{k}, B_{k}=D_{k}, k=\overline{0, n}$, where $d_{k}, D_{k}$, are, respectively, the poles and circular domains of the quadratic differential

$$
Q(w) d w^{2}=-\frac{\left(n^{2}-\gamma\right) w^{n}+\gamma}{w^{2}\left(w^{n}-1\right)^{2}} d w^{2}
$$

Moreover, instead of $n_{0}$ we can take $\left[e^{\frac{1}{\left(\frac{2}{3}-\alpha\right)^{2}}}\right]+1$. 
Proof. For $0<\gamma \leq \sqrt[3]{n}$ the problem was solved in [21] for any $n \geq 5$. Using that $5 \leq e^{\frac{1}{\left(\frac{2}{3}-\frac{1}{3}\right)^{2}}}$ for $\gamma=\sqrt[3]{n}$ theorem is proved. Prove the validity of the theorem for the case $\gamma=n^{\delta}$ at fixed $\delta \in\left(\frac{1}{3}, \frac{2}{3}\right)$. Let $\zeta=\pi_{k}(w)$ denote a singlevalued branch of a multivalued analytic function $-i\left(e^{-i \arg a_{k}} w\right)^{\frac{1}{\alpha_{k}}}, k=\overline{1, n}$, that realizes a univalent and conformal mapping $\bar{\Gamma}_{k}$ onto the right half-plane $\operatorname{Re} \zeta>0$. Consider the system of functions

$$
\zeta=\pi_{k}(w)=-i\left(e^{-i \arg a_{k}} w\right)^{\frac{1}{\alpha_{k}}}, \quad k=\overline{1, n} .
$$

The family of functions $\left\{\pi_{k}(w)\right\}_{k=1}^{n}$ is called admissible for the separating transformation of the domains $B_{k}, k=\overline{0, n}$ relative to the angles $\left\{\Gamma_{k}\right\}_{k=1}^{n}$. Let $\Omega_{k}^{(1)}, k=\overline{1, n}$ denote a domain of the plane $\mathbb{C}_{\zeta}$ obtained as a result of the union of the connected component of the set $\pi_{k}\left(B_{k} \cap \bar{\Gamma}_{k}\right)$ containing the point $\pi_{k}\left(a_{k}\right)$ with the own symmetric reflection relative to the imaginary axis. By $\Omega_{k}^{(2)}, k=\overline{1, n}$ we denote the domain of the plane $\mathbb{C}_{\zeta}$ obtained as a result of the union of the connected component of the set $\pi_{k}\left(B_{k+1} \bigcap \bar{\Gamma}_{k}\right)$ containing the point $\pi_{k}\left(a_{k+1}\right)$ with the own symmetric reflection relative to the imaginary axis, $B_{n+1}:=B_{1}, \pi_{n}\left(a_{n+1}\right):=\pi_{n}\left(a_{1}\right)$. In addition, by $\Omega_{k}^{(0)}$ we denote the domain of the plane $\mathbb{C}_{\zeta}$ obtained as a result of the union of the connected component of the set $\pi_{k}\left(B_{0} \cap \bar{\Gamma}_{k}\right)$ containing the point $\zeta=0$ with the own symmetric reflection relative to the imaginary axis. We denote

$$
\pi_{k}\left(a_{k}\right):=\omega_{k}^{(1)}, \quad \pi_{k}\left(a_{k+1}\right):=\omega_{k}^{(2)}, \quad k=\overline{1, n} .
$$

The definition of the functions $\pi_{k}$ implies that

$$
\begin{gathered}
\left|\pi_{k}(w)-\omega_{k}^{(1)}\right| \sim \frac{1}{\alpha_{k}}\left|a_{k}\right|^{\frac{1}{\alpha_{k}}-1} \cdot\left|w-a_{k}\right|, \quad w \rightarrow a_{k}, \quad w \in \overline{\Gamma_{k}}, \\
\left|\pi_{k}(w)-\omega_{k}^{(2)}\right| \sim \frac{1}{\alpha_{k}}\left|a_{k+1}\right|^{\frac{1}{\alpha_{k}}-1} \cdot\left|w-a_{k+1}\right|, \quad w \rightarrow a_{k+1}, \quad w \in \overline{\Gamma_{k}}, \\
\left|\pi_{k}(w)\right| \sim|w|^{\frac{1}{\alpha_{k}}}, \quad w \rightarrow 0, \quad w \in \overline{\Gamma_{k}} .
\end{gathered}
$$

Using the corresponding results of works $[13,17]$ we have the inequalities

$$
\begin{aligned}
& r\left(B_{k}, a_{k}\right) \leq\left[\frac{r\left(\Omega_{k}^{(1)}, \omega_{k}^{(1)}\right) \cdot r\left(\Omega_{k-1}^{(2)}, \omega_{k-1}^{(2)}\right)}{\frac{1}{\alpha_{k}}\left|a_{k}\right|^{\frac{1}{\alpha_{k}}-1} \cdot \frac{1}{\alpha_{k-1}}\left|a_{k}\right|^{\frac{1}{\alpha_{k-1}}-1}}\right]^{\frac{1}{2}}, \quad k=\overline{1, n} \\
& r\left(B_{0}, 0\right) \leq\left[\prod_{k=1}^{n} r^{\alpha_{k}^{2}}\left(\Omega_{k}^{(0)}, 0\right)\right]^{\frac{1}{2}} .
\end{aligned}
$$


Inequalities (3) and (4) yield the relation

$$
\begin{gathered}
r^{\gamma}\left(B_{0}, 0\right) \prod_{k=1}^{n} r\left(B_{k}, a_{k}\right) \leq \\
\leq \prod_{k=1}^{n} \alpha_{k} \prod_{k=1}^{n} \frac{\left|a_{k}\right|}{\left|a_{k} a_{k+1}\right|^{\frac{1}{2 \alpha_{k}}}}\left[\prod_{k=1}^{n} r^{\gamma \alpha_{k}^{2}}\left(\Omega_{k}^{(0)}, 0\right) r\left(\Omega_{k}^{(1)}, \omega_{k}^{(1)}\right) r\left(\Omega_{k}^{(2)}, \omega_{k}^{(2)}\right)\right]^{\frac{1}{2}} .
\end{gathered}
$$

It is known that the following functional

$$
\begin{gathered}
Y_{3}\left(t_{1}, t_{2}, t_{3}, D_{1}, D_{2}, D_{3}, d_{1}, d_{2}, d_{3}\right)= \\
=\frac{r^{t_{1}}\left(D_{1}, d_{1}\right) \cdot r^{t_{2}}\left(D_{2}, d_{2}\right) \cdot r^{t_{3}}\left(D_{3}, d_{3}\right)}{\left|d_{1}-d_{2}\right|^{t_{1}+t_{2}-t_{3}} \cdot\left|d_{1}-d_{3}\right|^{t_{1}-t_{2}+t_{3}} \cdot\left|d_{2}-d_{3}\right|^{-t_{1}+t_{2}+t_{3}}}
\end{gathered}
$$

is invariant with respect to all conformal automorphisms of the extended complex plane $\overline{\mathbb{C}}, t_{k} \in \mathbb{R}^{+},\left\{D_{k}\right\}_{k=1}^{3}$ is any system of non-overlapping domains such that $d_{k} \in D_{k} \subset \overline{\mathbb{C}}, k=1,2,3$.

Using formulae (5) and (6) after simple calculations we obtain

$$
\begin{gathered}
I_{n}(\gamma) \leq\left(\prod_{k=1}^{n} \alpha_{k}\right) \cdot \prod_{k=1}^{n} \frac{\left|a_{k}\right|}{\left|a_{k} a_{k+1}\right|^{\frac{1}{2 \alpha_{k}}}} \times \\
\times\left\{\prod_{k=1}^{n} \frac{r^{\gamma \alpha_{k}^{2}}\left(\Omega_{k}^{(0)}, 0\right) \cdot r\left(\Omega_{k}^{(1)}, \omega_{k}^{(1)}\right) \cdot r\left(\Omega_{k}^{(2)}, \omega_{k}^{(2)}\right)}{\left|\omega_{k}^{(1)} \cdot \omega_{k}^{(2)}\right| \gamma \alpha_{k}^{2}\left|\omega_{k}^{(1)}-\omega_{k}^{(2)}\right|^{2-\gamma \alpha_{k}^{2}}}\right\}^{\frac{1}{2}} \times \\
\times\left[\prod_{k=1}^{n}\left|\omega_{k}^{(1)} \cdot \omega_{k}^{(2)}\right| \gamma \alpha_{k}^{2}\left|\omega_{k}^{(1)}-\omega_{k}^{(2)}\right|^{2-\gamma \alpha_{k}^{2}}\right]^{\frac{1}{2}},
\end{gathered}
$$

where $\left|\omega_{k}^{(1)}\right|=\left|a_{k}\right|^{\frac{1}{\alpha_{k}}},\left|\omega_{k}^{(2)}\right|=\left|a_{k+1}\right|^{\frac{1}{\alpha_{k}}},\left|\omega_{k}^{(1)}-\omega_{k}^{(2)}\right|=\left|a_{k}\right|^{\frac{1}{\alpha_{k}}}+\left|a_{k+1}\right|^{\frac{1}{\alpha_{k}}}$, $k=\overline{1, n}$. Thus summing the above relations we obtain

$$
\begin{gathered}
I_{n}(\gamma) \leq 2^{n-\frac{\gamma}{2}} \sum_{k=1}^{n} \alpha_{k}^{2} \cdot\left(\prod_{k=1}^{n} \alpha_{k}\right) \cdot \prod_{k=1}^{n}\left[\chi\left(\left|\frac{a_{k}}{a_{k+1}}\right|^{\frac{1}{2 \alpha_{k}}}\right)\right]^{1-\frac{\gamma \alpha_{k}^{2}}{2}} \times \\
\times \prod_{k=1}^{n}\left|a_{k}\right|^{1+\frac{1}{4} \gamma\left(\alpha_{k}+\alpha_{k-1}\right)} \cdot\left\{\prod_{k=1}^{n} Y_{3}\left(\gamma \alpha_{k}^{2}, 1,1, \Omega_{k}^{(0)}, \Omega_{k}^{(1)}, \Omega_{k}^{(2)}, 0, \omega_{k}^{(1)}, \omega_{k}^{(2)}\right)\right\}^{\frac{1}{2}} .
\end{gathered}
$$


It is clear that for any $k=\overline{1, n}$ we can indicate conformal automorphism $\zeta=T_{k}(z)$ of the plane of complex numbers $\overline{\mathbb{C}}$ such that $T_{k}(0)=0, T_{k}\left(\omega_{k}^{(s)}\right)=$ $(-1)^{s} \cdot i, D_{k}^{(q)}:=T_{k}\left(\Omega_{k}^{(q)}\right), k=\overline{1, n}, s=1,2, q=0,1,2$. Under condition of conformal invariance of the functional (7) we obtain

$$
\begin{aligned}
& Y_{3}\left(\gamma \alpha_{k}^{2}, 1,1, \Omega_{k}^{(0)}, \Omega_{k}^{(1)}, \Omega_{k}^{(2)}, 0, \omega_{k}^{(1)}, \omega_{k}^{(2)}\right)= \\
& \quad=Y_{3}\left(\gamma \alpha_{k}^{2}, 1,1, D_{k}^{(0)}, D_{k}^{(1)}, D_{k}^{(2)}, 0,-i, i\right),
\end{aligned}
$$

where $k=\overline{1, n}$ and

$$
\begin{gathered}
Y_{3}\left(\gamma \alpha_{k}^{2}, 1,1, D_{k}^{(0)}, D_{k}^{(1)}, D_{k}^{(2)}, 0,-i, i\right)= \\
=\frac{r^{\alpha_{k}^{2} \gamma}\left(D_{k}^{(0)}, 0\right) \cdot r\left(D_{k}^{(1)},-i\right) \cdot r\left(D_{k}^{(2)}, i\right)}{2^{2-\gamma \alpha_{k}^{2}}} .
\end{gathered}
$$

Hence

$$
\begin{aligned}
& I_{n}(\gamma) \leq 2^{n-\frac{\gamma}{2} \sum_{k=1}^{n} \alpha_{k}^{2}} \cdot\left(\prod_{k=1}^{n} \alpha_{k}\right) \cdot \mathcal{K}^{(\gamma)}\left(A_{n}\right) \times \\
& \times\left\{\prod_{k=1}^{n} \frac{r^{\alpha_{k}^{2} \gamma}\left(D_{k}^{(0)}, 0\right) \cdot r\left(D_{k}^{(1)},-i\right) \cdot r\left(D_{k}^{(2)}, i\right)}{2^{2-\gamma \alpha_{k}^{2}}}\right\}^{\frac{1}{2}} .
\end{aligned}
$$

Taking last condition and condition (8) into account we obtain the following estimate

$$
\begin{gathered}
I_{n}(\gamma) \leq 2^{n-\frac{\gamma}{2} \sum_{k=1}^{n} \alpha_{k}^{2}}\left(\prod_{k=1}^{n} \alpha_{k}\right) \cdot \mathcal{K}^{(\gamma)}\left(A_{n}\right) \cdot 2^{-n+\frac{\gamma}{2} \sum_{k=1}^{n} \alpha_{k}^{2}} \times \\
\times\left[\prod_{k=1}^{n} r^{\alpha_{k}^{2} \gamma}\left(D_{k}^{(0)}, 0\right) r\left(D_{k}^{(1)},-i\right) r\left(D_{k}^{(2)}, i\right)\right]^{\frac{1}{2}} .
\end{gathered}
$$

Taking into account the conditions of the theorem we obtain the inequality

$$
I_{n}(\gamma) \leq\left(\prod_{k=1}^{n} \alpha_{k}\right)\left[\prod_{k=1}^{n} r^{\alpha_{k}^{2} \gamma}\left(D_{k}^{(0)}, 0\right) r\left(D_{k}^{(1)},-i\right) r\left(D_{k}^{(2)}, i\right)\right]^{\frac{1}{2}}
$$

where $D_{k}^{(0)}, D_{k}^{(1)}, D_{k}^{(2)}$ are circular domains of the quadratic differential

$$
Q(w) d w^{2}=\frac{\left(4-\alpha_{k}^{2} \gamma\right) w^{2}-\alpha_{k}^{2} \gamma}{w^{2}\left(w^{2}+1\right)^{2}} d w^{2}
$$


$\left(0 \in D_{k}^{(0)},-i \in D_{k}^{(1)}, i \in D_{k}^{(2)}\right)$. Using paper [17], from (9), we have

$$
\begin{gathered}
I_{n}(\gamma) \leq \\
\leq\left(\prod_{k=1}^{n} \alpha_{k}\right)\left[\prod_{k=1}^{n} 2^{\tau_{k}^{2}+6} \cdot \tau_{k}^{\tau_{k}^{2}} \cdot\left(2-\tau_{k}\right)^{-\frac{1}{2}\left(2-\tau_{k}\right)^{2}} \cdot\left(2+\tau_{k}\right)^{-\frac{1}{2}\left(2+\tau_{k}\right)^{2}}\right]^{1 / 2},
\end{gathered}
$$

where $\tau_{k}=\alpha_{k} \sqrt{\gamma}, k=\overline{1, n}$.

Note, that in [19] the problem was completely solved if $n \geq 5$ and $\alpha_{k} \sqrt{\gamma} \leq$ 2. Thus we have to prove its to the case $\alpha_{0} \sqrt{\gamma}>2$, where $\alpha_{0}=\max _{k} \alpha_{k}$. Thus consider the case when

$$
\alpha_{0} \sqrt{\gamma} \geq 2, \quad \alpha_{0}=\max _{k} \alpha_{k}
$$

The following equality holds

$$
I_{n}(\gamma)=\prod_{k=1}^{n}\left[r\left(B_{0}, 0\right) r\left(B_{k}, a_{k}\right)\right]^{\frac{\gamma}{n}}\left[\prod_{k=1}^{n} r\left(B_{k}, a_{k}\right)\right]^{1-\frac{\gamma}{n}} .
$$

From Lavrent'ev's theorem [5] it follows that

$$
r\left(B_{0}, 0\right) r\left(B_{k}, a_{k}\right) \leq\left|a_{k}\right|^{2} .
$$

Then

$$
I_{n}(\gamma) \leq \prod_{k=1}^{n}\left|a_{k}\right|^{\frac{2 \gamma}{n}}\left[\prod_{k=1}^{n} r\left(B_{k}, a_{k}\right)\right]^{1-\frac{\gamma}{n}} .
$$

From $\mathcal{K}^{(0)}\left(A_{n}\right) \leq 1$ we have $\prod_{k=1}^{n}\left|a_{k}\right| \leq 1$, then

$$
I_{n}(\gamma) \leq\left[\prod_{k=1}^{n} r\left(B_{k}, a_{k}\right)\right]^{1-\frac{\gamma}{n}} .
$$

From Theorem 5.1.1 [13]

$$
\prod_{k=1}^{n} r\left(B_{k}, a_{k}\right) \leq 2^{n} \prod_{k=1}^{n} \alpha_{k} \cdot \mathcal{K}^{(0)}\left(A_{n}\right)
$$

Therefore

$$
I_{n}(\gamma) \leq\left[2^{n} \cdot \prod_{k=1}^{n} \alpha_{k}\right]^{1-\frac{\gamma}{n}}
$$


Since $\sum_{k=1}^{n} \alpha_{k}=2$ then

$$
\prod_{k=1}^{n} \alpha_{k} \leq \alpha_{0} \prod_{k=1, k \neq k_{0}}^{n} \alpha_{k} \leq \alpha_{0}\left(\frac{\sum_{k=1, k \neq k_{0}}^{n} \alpha_{k}}{n-1}\right)^{n-1}=\alpha_{0}\left(\frac{2-\alpha_{0}}{n-1}\right)^{n-1},
$$

where $\alpha_{0}:=\alpha_{k_{0}}:=\max _{1 \leq k \leq n} \alpha_{k}$. Thus, from (11) we have

$$
I_{n}(\gamma) \leq\left[2^{n} \alpha_{0}\left(2-\alpha_{0}\right)^{n-1}(n-1)^{-(n-1)}\right]^{1-\frac{\gamma}{n}} .
$$

Let

$$
I_{n}^{0}(\gamma)=r^{\gamma}\left(D_{0}, 0\right) \prod_{k=1}^{n} r\left(D_{k}, d_{k}\right),
$$

where $0 \cup\left\{d_{k}\right\}_{k=1}^{n}$ and $\left\{D_{k}\right\}_{k=0}^{n}$ are, respectively, the poles and circular domains of the quadratic differential (2). From [13, 17, 19] taking into account (10) we obtain

$$
I_{n}^{0}(\gamma)=\left(\frac{4}{n}\right)^{n} \frac{\left(\frac{4 \gamma}{n^{2}}\right)^{\frac{\gamma}{n}}}{\left(1-\frac{\gamma}{n^{2}}\right)^{n+\frac{\gamma}{n}}}\left(\frac{1-\frac{\sqrt{\gamma}}{n}}{1+\frac{\sqrt{\gamma}}{n}}\right)^{2 \sqrt{\gamma}}
$$

Let

$$
P_{n}(\gamma):=\frac{I_{n}(\gamma)}{I_{n}^{0}(\gamma)}
$$

Then

$$
\begin{gathered}
P_{n}(\gamma) \leq \frac{\left[2 \cdot 2^{n-1} \cdot \alpha_{0}\left(2-\alpha_{0}\right)^{n-1}(n-1)^{-(n-1)}\right]^{1-\frac{\gamma}{n}}}{\left(\frac{4}{n}\right)^{n-1-\gamma\left(1-\frac{1}{n}\right)}\left(\frac{4}{n}\right)^{\gamma+1-\frac{\gamma}{n}}\left(\frac{4 \gamma}{n^{2}}\right)^{\frac{\gamma}{n}}\left(1-\frac{\gamma}{n^{2}}\right)^{-n-\frac{\gamma}{n}}\left(\frac{1-\frac{\sqrt{\gamma}}{n}}{1+\frac{\sqrt{\gamma}}{n}}\right)^{2 \sqrt{\gamma}}}, \\
\alpha_{0} \geq \frac{2}{\sqrt{\gamma}}>\frac{2}{n} .
\end{gathered}
$$

So,

$$
\begin{aligned}
P_{n}(\gamma) & \leq\left[\frac{n}{4}\right]^{\gamma+1}\left[1-\frac{1}{\sqrt{\gamma}}\right]^{n-1-\gamma \frac{n-1}{n}}\left(\frac{n}{\gamma}\right)^{\frac{\gamma}{n}}\left(1-\frac{\gamma}{n^{2}}\right)^{n+\frac{\gamma}{n}} \times \\
& \times\left(\frac{1+\frac{\sqrt{\gamma}}{n}}{1-\frac{\sqrt{\gamma}}{n}}\right)^{2 \sqrt{\gamma}}\left(\frac{4}{\sqrt{\gamma}}\right)^{1-\frac{\gamma}{n}}\left(\frac{n}{n-1}\right)^{n-1-\gamma \frac{n-1}{n}} .
\end{aligned}
$$


Hence

$$
\begin{aligned}
P_{n}\left(n^{\delta}\right) \leq & {\left[\frac{n}{4}\right]^{n^{\delta}+1}\left[1-\frac{1}{n^{\frac{\delta}{2}}}\right]^{n-1-\frac{n-1}{n^{1-\delta}}}\left(n^{1-\delta}\right)^{\frac{1}{n^{1-\delta}}}\left(1-\frac{1}{n^{2-\delta}}\right)^{n+\frac{1}{n^{1-\delta}}} \times } \\
& \times\left(\frac{1+\frac{1}{n^{1-\frac{\delta}{2}}}}{1-\frac{1}{n^{1-\frac{\delta}{2}}}}\right)^{2 n^{\frac{\delta}{2}}}\left(\frac{4}{n^{\frac{\delta}{2}}}\right)^{1-\frac{1}{n^{1-\delta}}}\left(\frac{n}{n-1}\right)^{n-1-\frac{n-1}{n^{1-\delta}}} .
\end{aligned}
$$

Let

$$
\begin{gathered}
g(x)=\left[\frac{x}{4}\right]^{x^{\delta}+1} \cdot\left[1-\frac{1}{x^{\frac{\delta}{2}}}\right]^{x-1-\frac{x-1}{x^{1-\delta}}}, \quad f_{1}(x)=\left(x^{1-\delta}\right)^{\frac{1}{x^{1-\delta}}}, \\
f_{2}(x)=\left(1-\frac{1}{x^{2-\delta}}\right)^{x+\frac{1}{x^{1-\delta}}}, \quad f_{3}(x)=\left(\frac{1+\frac{1}{x^{1-\frac{\delta}{2}}}}{1-\frac{1}{x^{1-\frac{\delta}{2}}}}\right)^{2 x^{\frac{\delta}{2}}}, \\
f_{4}(x)=\left(\frac{4}{x^{\frac{\delta}{2}}}\right)^{1-\frac{1}{x^{1-\delta}}}, \quad f_{5}(x)=\left(\frac{x}{x-1}\right)^{x-1-\frac{x-1}{x^{1-\delta}}} .
\end{gathered}
$$

Explore these functions for $x \in\left[e^{9} ; \infty\right)$. For $g(x)$ we obtain

$$
\begin{gathered}
\ln (g(x))=\left(x^{\delta}+1\right) \ln \frac{x}{4}+\left(x-1-x^{\delta}+x^{\delta-1}\right) \ln \left(1-\frac{1}{x^{\frac{\delta}{2}}}\right) . \\
(\ln (g(x)))^{\prime}=\left(\delta x^{\delta-1}\right) \ln \frac{x}{4}+x^{\delta-1}+\frac{1}{x}+\left(1-\delta x^{\delta-1}+(\delta-1) x^{\delta-2}\right) \ln \left(1-\frac{1}{x^{\frac{\delta}{2}}}\right)+ \\
+\frac{1}{1-\frac{1}{x^{\frac{\delta}{2}}}} \cdot \frac{\delta}{2} \frac{1}{x^{1+\frac{\delta}{2}}}\left(x-1-x^{\delta}+x^{\delta-1}\right) .
\end{gathered}
$$

Since $x \geq e^{9}$ then the following inequalities hold

$$
\begin{gathered}
\ln \left(1-\frac{1}{x^{\frac{\delta}{2}}}\right)=-\frac{1}{x^{\frac{\delta}{2}}}-\frac{1}{2 x^{\delta}}-\frac{1}{3 x^{\frac{3 \delta}{2}}}-\ldots \leq-\frac{1}{x^{\frac{\delta}{2}}}-\frac{1}{2 x^{\delta}} \\
\frac{1}{1-\frac{1}{x^{\frac{\delta}{2}}}} \leq \frac{1}{1-\frac{1}{\left(e^{9}\right)^{\frac{1}{6}}}}<1,3, \quad\left(x-1-x^{\delta}+x^{\delta-1}\right)>0, \\
\left(1-\delta x^{\delta-1}+(\delta-1) x^{\delta-2}\right)>0 .
\end{gathered}
$$

Prove that for $x \geq e^{\frac{1}{\left(\frac{2}{3}-\delta\right)^{2}}}$ and fixed $\delta$ the inequality holds

$$
\ln x \leq x^{\frac{2}{3}-\delta} .
$$


Consider the function $h(x)=x^{\frac{2}{3}-\delta}-\ln x$ for $x \geq e^{\frac{1}{\left(\frac{2}{3}-\delta\right)^{2}}}$. Thus

$$
(h(x))^{\prime}=\frac{1}{x}\left(\left(\frac{2}{3}-\delta\right) x^{\frac{2}{3}-\delta}-1\right) .
$$

On the other hand

$$
\left(\frac{2}{3}-\delta\right) x^{\frac{2}{3}-\delta}-1 \geq\left(\frac{2}{3}-\delta\right)\left(\frac{1}{\frac{2}{3}-\delta}+\frac{1}{2\left(\frac{2}{3}-\delta\right)^{2}}\right)-1=\frac{1}{2\left(\frac{2}{3}-\delta\right)}>0 .
$$

Thus, the function $h(x)$ is monotonically increasing for $x \in\left[e^{\frac{1}{\left(\frac{2}{3}-\delta\right)^{2}}} ;+\infty\right)$ and

$$
h\left(e^{\overline{\left(\frac{2}{3}-\delta\right)^{2}}}\right)=e^{\frac{1}{\left(\frac{2}{3}-\delta\right)}}-\frac{1}{\left(\frac{2}{3}-\delta\right)^{2}}>0 .
$$

It follows that $\ln x \leq x^{\frac{2}{3}-\delta}$. Taking last inequalities into account we obtain

$$
\begin{aligned}
(\ln (g(x)))^{\prime}<\frac{\delta}{x^{\frac{1}{3}}}+ & \frac{1-\delta \ln 4}{x^{1-\delta}}-\frac{1-0,65 \delta}{x^{\frac{\delta}{2}}}-\frac{1}{2 x^{\delta}}+\frac{0,35 \delta}{x^{1-\frac{\delta}{2}}}+\frac{\frac{\delta}{2}+1}{x}- \\
& -\frac{0,65 \delta}{x^{1+\frac{\delta}{2}}}+\frac{1-0,35 \delta}{x^{2-\frac{\delta}{2}}}+\frac{1-\delta}{x^{2}} .
\end{aligned}
$$

Denote

$$
\begin{gathered}
A(x)=\frac{\delta}{x^{\frac{1}{3}}}+\frac{1-\delta \ln 4}{x^{1-\delta}}-\frac{1-0,65 \delta}{x^{\frac{\delta}{2}}}, \\
B(x)=-\frac{1}{2 x^{\delta}}+\frac{0,35 \delta}{x^{1-\frac{\delta}{2}}}+\frac{\frac{\delta}{2}+1}{x}, \\
C(x)=-\frac{0,65 \delta}{x^{1+\frac{\delta}{2}}}+\frac{1-0,35 \delta}{x^{2-\frac{\delta}{2}}}+\frac{1-\delta}{x^{2}} .
\end{gathered}
$$

Using the standard methods of researching the functions for fixed $\delta$ and $x \in$ $\left[e^{\frac{1}{\left(\frac{2}{3}-\delta\right)^{2}}} ;+\infty\right)$ we have that

$$
A(x)=\frac{1}{x^{\frac{\delta}{2}}}\left(-(1-0,65 \delta)+\frac{\delta}{x^{\frac{1}{3}-\frac{\delta}{2}}}+\frac{1-\delta \ln 4}{x^{1-\frac{3 \delta}{2}}}\right)
$$

and

$$
-(1-0,65 \delta)+\frac{\delta}{x^{\frac{1}{3}-\frac{\delta}{2}}}+\frac{1-\delta \ln 4}{x^{1-\frac{3 \delta}{2}}}<-0,5+\frac{\frac{2}{3}}{e^{\frac{1}{2\left(\frac{2}{3}-\delta\right)}}}+\frac{0,6}{e^{\frac{3}{2\left(\frac{2}{3}-\delta\right)}}<}<
$$




$$
<-0,5+0,15+0,01=-0,34<0 .
$$

Thus $A(x)<0$ for all $x$. Similarly,

$$
\begin{aligned}
& B(x)=-\frac{0,3}{x^{\delta}}+\frac{0,35 \delta}{x^{1-\frac{\delta}{2}}}-\frac{0,2}{x^{\delta}}+\frac{\frac{\delta}{2}+1}{x}<\left(-\frac{0,3}{x^{\delta}}+\frac{0,3}{x^{1-\frac{\delta}{2}}}\right)+\left(-\frac{0,2}{x^{\delta}}+\frac{1,4}{x}\right)<0 . \\
& C(x)=\frac{1}{x^{1+\frac{\delta}{2}}}\left(-0,65 \delta+\frac{1-0,35 \delta}{x^{1-\delta}}+\frac{1-\delta}{x^{1-\frac{\delta}{2}}}\right)<\frac{1}{x^{1+\frac{\delta}{2}}}\left(-0,2+\frac{1}{x^{\frac{1}{3}}}+\frac{1}{x^{\frac{2}{3}}}\right)<0 .
\end{aligned}
$$

Since $(\ln (g(x)))^{\prime}<A(x)+B(x)+C(x)$ then $(\ln (g(x)))^{\prime}<0$. Thereby, the function $g(x)$ is monotonically decreasing for fixed $\delta$ and $x \in\left[e^{\frac{1}{\left(\frac{2}{3}-\delta\right)^{2}}} ;+\infty\right)$.

Consider $f_{1}(x)$. It is not difficult to obtain

$$
\ln f_{1}(x)=\left(\frac{1}{x^{1-\delta}}\right) \ln \left(x^{1-\delta}\right)
$$

Thus we have

$$
\begin{gathered}
\left(\ln \left(x^{1-\delta}\right)^{\frac{1}{x^{1-\delta}}}\right)^{\prime}=\left(\frac{1}{x^{1-\delta}} \ln \left(x^{1-\delta}\right)\right)^{\prime}=(1-\delta)\left(\frac{1}{x^{1-\delta}} \ln x\right)^{\prime}= \\
=(1-\delta)\left(-(1-\delta) x^{-(2-\delta)} \ln x+x^{-(2-\delta)}\right)=(1-\delta) x^{-(2-\delta)}(-(1-\delta) \ln x+1) .
\end{gathered}
$$

Hence $\left(\ln f_{1}(x)\right)^{\prime}=(-(1-\delta) \ln x+1)<0$ and $x>e^{\frac{1}{1-\delta}}$. Since $e^{\frac{1}{1-\delta}}$ for $\frac{1}{3}<\delta<\frac{2}{3}$ does not exceed $e^{\frac{1}{1-\frac{2}{3}}}=e^{3}<21$, then for $x \in\left[e^{9} ;+\infty\right]$ the function $f_{1}(x)$ is monotonically decreasing. Thus for any $n \geq e^{9}$ the following inequality holds

$$
\left(x^{1-\delta}\right)^{\frac{1}{x^{1-\delta}}} \leq\left(\left(e^{9}\right)^{\frac{1}{3}}\right)^{\frac{1}{\left(e^{9}\right)^{\frac{1}{3}}}} \leq 1,06
$$

It's clear that $\left(1-\frac{1}{x^{2-\delta}}\right)<1$ and therefore $\left(1-\frac{1}{x^{2-\delta}}\right)^{x+\frac{1}{x^{1-\delta}}}<1$. That is for any $x \geq e^{9}$ we obtain

$$
f_{2}(x)<1 \text {. }
$$

Consider $f_{3}(x)$.

$$
\ln f_{3}(x)=2 x^{\frac{\delta}{2}} \ln \left(1+\frac{1}{x^{1-\frac{\delta}{2}}}\right)+2 x^{\frac{\delta}{2}} \ln \left(1-\frac{1}{x^{1-\frac{\delta}{2}}}\right)
$$

and thus

$$
\left(\ln f_{3}(x)\right)^{\prime}=\delta x^{\frac{\delta}{2}-1} \ln \left(1+\frac{2}{x^{1-\frac{\delta}{2}}}\right)-4\left(1-\frac{\delta}{2}\right) x^{\delta-2} \cdot \frac{1}{x^{2-\delta}-1} \leq 0
$$


So, for $x \geq e^{9}$ the function $f_{3}(x)$ is monotonically decreasing and for any $n \geq e^{9}$ we have

$$
\left(\frac{1+\frac{1}{n^{1-\frac{\delta}{2}}}}{1-\frac{1}{n^{1-\frac{\delta}{2}}}}\right)^{2 n^{\frac{\delta}{2}}} \leq\left(\frac{1+\frac{1}{\left(e^{9}\right)^{\frac{2}{3}}}}{1-\frac{1}{\left(e^{9}\right)^{\frac{2}{3}}}}\right)^{2 \cdot\left(e^{9}\right)^{\frac{1}{3}}} \leq 1,22 .
$$

As for $f_{4}(x)$ it is clear

$$
\left(\frac{4}{x^{\frac{\delta}{2}}}\right)^{1-\frac{1}{x^{1-\delta}}} \leq\left(\frac{4}{\left(e^{9}\right)^{\frac{1}{6}}}\right)^{1-\frac{1}{\left(e^{9}\right)^{\frac{1}{3}}}} \leq 1 .
$$

Thus, the function $f_{4}(x)$ on the interval $\left[e^{9} ;+\infty\right)$ is monotonically decreasing and for any $n \geq e^{9}$ we obtain

$$
\left(\frac{4}{x^{\frac{\delta}{2}}}\right)^{1-\frac{1}{x^{1-\delta}}} \leq 1
$$

Since $\frac{x}{x-1}>1$ for any $x \in\left[e^{9} ;+\infty\right)$, then

$$
f_{5}(x)=\left(\frac{x}{x-1}\right)^{x-1-\frac{x-1}{x^{1-\delta}}} \leq\left(1+\frac{1}{x-1}\right)^{x-1} .
$$

The function $\left(1+\frac{1}{x-1}\right)^{x-1}$ is monotonically increasing on the interval $\left[e^{9} ;+\infty\right)$ and

$$
\lim _{x \rightarrow \infty}\left(1+\frac{1}{x-1}\right)^{x-1}=e
$$

thus for any $n \geq e^{9}$

$$
\left(\frac{n}{n-1}\right)^{n-1-\frac{n-1}{n^{1-\delta}}} \leq e
$$

Taking properties of the investigated functions $f_{j}(x), j=\overline{1,5}$ into account we obtain

$$
P_{n}\left(n^{\delta}\right)=g(x) \prod_{j=1}^{5} f_{j}(n)<g(x) \cdot 1,06 \cdot 1 \cdot 1,22 \cdot 1 \cdot e<5 g(x) .
$$

Since $g(x)<0,1$ for $x=e^{\frac{1}{\left(\frac{2}{3}-\delta\right)^{2}}}$, therefore

$$
P_{n}(\gamma) \leq 0,5<1
$$


Let $\gamma \in\left(1, \gamma_{n}\right]$. Taking into account that the function

$$
\left[2^{n} \cdot \frac{2}{\sqrt{\gamma}}\left(2-\frac{2}{\sqrt{\gamma}}\right)^{n-1}(n-1)^{-(n-1)}\right]^{1-\frac{\gamma}{n}}
$$

for fixed $n$ increases monotonically with respect to $\gamma$ on the interval $\left(1, \gamma_{n}\right]$, and function $I_{n}^{0}(\gamma)$ monotonically decreases with respect to $\gamma$ on the same interval, since

$$
\left(\ln I_{n}^{0}(\gamma)\right)^{\prime}=\left(\frac{1}{n} \ln \left(\frac{4 \gamma}{n^{2}-\gamma}\right)+\frac{1}{\sqrt{\gamma}} \ln \left(\frac{n-\sqrt{\gamma}}{n+\sqrt{\gamma}}\right)\right)<0
$$

for each fixed $n$. Then we get that

$$
P_{n}(\gamma)=\frac{I_{n}(\gamma)}{I_{n}^{0}(\gamma)}<\frac{I_{n}\left(\gamma_{n}\right)}{I_{n}^{0}\left(\gamma_{n}\right)}=P_{n}\left(\gamma_{n}\right)<1
$$

Thus, at these parameter values, extremal values of the functional $I_{n}(\gamma)$ are not achieved. For $\gamma \in\left(1, \gamma_{n}\right], \alpha_{0} \sqrt{\gamma} \geq 2, n \geq 2$, the following inequality holds

$$
I_{n}(\gamma)<I_{n}^{0}(\gamma) \text {. }
$$

It follows that the configuration written in the hypothesis of the Theorem 1 is extremal. So, we have to investigate the case $\alpha_{0} \sqrt{\gamma}<2$. Using the results of the papers [19, 26, 27], we obtain the validity of the Theorem 1 and for this case too.

The Theorem 1 can be obtained in another simpler form. Let $\delta=\frac{2}{3}-\frac{1}{\sqrt{\ln n}}$. Then we obtain that $n=e^{\frac{1}{\left(\frac{2}{3}-\delta\right)^{2}}}$. And the following statement is true.

Theorem 2. Let $n \in \mathbb{N}, n \geq e^{9}, \gamma \in\left(0, \gamma_{n}\right], \gamma_{n}=n^{\frac{2}{3}-\frac{1}{\sqrt{\ln n}}}$. Then for any $n$-radial system of points $A_{n}=\left\{a_{k}\right\}_{k=1}^{n}$ such that $\mathcal{K}^{(\gamma)}\left(A_{n}\right) \leq 1, \mathcal{K}^{(0)}\left(A_{n}\right) \leq$ 1 , and any system of pairwise non-overlapping domains $B_{k}, a_{k} \in B_{k} \subset \overline{\mathbb{C}}$, $a_{0}=0 \in B_{0} \subset \overline{\mathbb{C}}(k=\overline{1, n})$, the inequality (1) holds. Equality is attained in the same case as in Theorem 1.

For arbitrary $\delta \geq \frac{2}{3}$ we can not apply the method used in the Theorem 1 . Because under this condition $\lim P_{n}(\gamma)=+\infty$ at $n \rightarrow \infty$ therefore, we can not find extremal configurations of the domains.

From Theorem 1 we obtain the following result.

Theorem 3. Under the conditions of Theorem 1, the following inequality holds

$$
r^{\gamma}\left(B_{0}, 0\right) \prod_{k=1}^{n} r\left(B_{k}, a_{k}\right) \leq\left(\frac{4}{n}\right)^{n} \frac{\left(\frac{4 \gamma}{n^{2}}\right)^{\frac{\gamma}{n}}}{\left(1-\frac{\gamma}{n^{2}}\right)^{n+\frac{\gamma}{n}}}\left(\frac{1-\frac{\sqrt{\gamma}}{n}}{1+\frac{\sqrt{\gamma}}{n}}\right)^{2 \sqrt{\gamma}} .
$$


Equality in inequality (12) is attained when points $a_{k}$ and domains $B_{k}, k=$ $\overline{0, n}$, are, respectively, the poles and circular domains of the quadratic differential (2).

\section{References}

[1] B. Riemann, Theorie der Abelschen Functionen, J. Reine Angew. Math., 54 (1867), 101-155.

[2] L. Bieberbach, Über die Koeffizienten derjenigen Potenzreihen, welche eine schlichte Abbildung des Einheitskreises vermitteln, Sitzungsber. Preuss. Akad. Wiss. Phys-Math. Kl., 138 (1916), 940-955.

[3] H. Grötzsch, Über einige Extremalprobleme der konformen Abbildung. I, II, Ber. Verh. Sächs. Akad. Wiss. Leipzig, Math.-Phys., 80(6) (1928), 367$376,497-502$.

[4] O. Teichmüller, Collected Papers, Springer, Berlin, 1982.

[5] M.A. Lavrent'ev, On the theory of conformal mappings, Tr. Sci. Inst An USSR, 5 (1934), 159-245. (in Russian)

[6] G.M. Goluzin, Geometric theory of functions of a complex variable, Amer. Math. Soc. Providence, RI, 1969.

[7] J. Jenkins, Univalent Functions and Conformal Mapping, Springer Verlag, Berlin-Göttingen-Heidelberg, 1958.

[8] Z. Nehari, Some inequalities in the theory of functions, Trans. Amer. Math. Soc., 75(2) (1953), 256-286.

[9] N.A. Lebedev, The area principle in the theory of univalent functions, Moscow: Science, 1975. (in Russian)

[10] V. Hayman, Multivalent functions, Cambridge University Press, Cambridge, 1958.

[11] P.L. Duren, M. Schiffer, Conformal mappings onto non-overlapping regions, Complex analysis. Basel: Birkhauser Verlag, (1988) 27-39.

[12] R. Kühnau, Über zwei Klassen schlichter konformer Abbildungen, Math. Nachr., 49, No. 1-6, (1971) 173-185. 
[13] A.K. Bakhtin, G.P. Bakhtina, Yu.B.Zelinskii, Topological-algebraic structures and geometric methods in complex analysis, Zb. prats of the Inst. of Math. of NASU, 2008. (in Russian)

[14] V.N. Dubinin, Condenser capacities and symmetrization in geometric function theory, Birkhäuser/Springer, Basel, 2014.

[15] G.V. Kuzmina, Methods of geometric function theory, II, St. Petersbg. Math. J., 5 (1997), 889-930.

[16] P.M. Tamrazov, Extremal conformal mappings and poles of quadratic differentials, Mathematics of the USSR-Izvestiya, 2(5) (1968), 987-996.

[17] V.N.Dubinin, Symmetrization method in geometric function theory of complex variables, Russian Math. Surveys, 1 (1994), 1-79.

[18] K. Strebel, Quadratic differentials, Springer-Verlag, 1984.

[19] L.V. Kovalev, On the problem of extremal decomposition with free poles on a circle, Dalnevostochnyi Mat. Sb. 2 (1996), 96-98. (in Russian)

[20] Ja.V. Zabolotnij, Some application of the method of separating transformation in one problem on nonoverlapping domains, Dopov. Nac. akad. nauk Ukr., (9) (2011), 13-17. (in Ukrainian)

[21] I.V. Denega, Quadratic differentials and a separating transformation in extremal problems on non-overlapping domains, Dopov. Nac. akad. nauk Ukr., (4) (2012), 15-19. (in Russian)

[22] A. Targonskii, Extremal problems on the generalized $(n, d)$-equiangular system of points, An. St. Univ. Ovidius Constanta, Ser. Mat., 22(2) (2014), 239-251.

[23] Ya. Zabolotnii, I. Dvorak, Some evaluation of maximum of the product of conformal radii for pairwise non-overlapping domains, Lobachevskii Journal of Mathematics, 38(3) (2017), 554-559.

[24] A.K. Bakhtin, Ya.V. Zabolotnii, Estimates of a product of the inner radii of nonoverlapping domains, Journal of Mathematical Sciences, 221(5) (2017), 623-629.

[25] I.V. Denega, Ya.V. Zabolotnii, Estimates of products of inner radii of non-overlapping domains in the complex plane, Complex Variables and Elliptic Equations, 62(11) (2017), 1611-1618. 
[26] A.K. Bakhtin, L.V. Vygivska, I.V. Denega, Inequalities for the internal radii of non-overlapping domains, Journal of Mathematical Sciences, $220(5)$ (2017), 584-590.

[27] A. Bakhtin, L. Vygivska and I. Denega, N-radial systems of points and problems for non-overlapping domains, Lobachevskii Journal of Mathematics, 38(2) (2017), 229-235.

Iryna Denega,

Department of complex analysis and potential theory,

Institute of mathematics of the National Academy of Sciences of Ukraine,

Tereschenkivska st. 3, 01004 Kiev-4, Ukraine.

Email: iradenega@gmail.com

Yaroslav Zabolotnii,

Department of complex analysis and potential theory,

Institute of mathematics of the National Academy of Sciences of Ukraine,

Tereschenkivska st. 3, 01004 Kiev-4, Ukraine.

Email: yaroslavzabolotnii@gmail.com 